\title{
The effects of cognitive bias modification for attention and interpretation on the postoperative psychological resilience and quality of life of patients with pituitary adenoma: a randomized trial
}

\author{
Jiajia Zhang ${ }^{1,2}$, Xujuan $\mathrm{Xu}^{1,3}$, Ziheng Wang ${ }^{2,4}$, Shichen Jiang ${ }^{2}$ \\ ${ }^{1}$ School of Nursing, Nantong University, Nantong, China; ${ }^{2}$ Department of Neurosurgery, Affiliated Hospital of Nantong University, Nantong, \\ China; ${ }^{3}$ Nursing Department, Nantong University Affiliated Hospital, Nantong, China; ${ }^{4}$ Department of Clinical Biobank, Affiliated Hospital of \\ Nantong University, Nantong, China \\ Contributions: (I) Conception and design: J Zhang, X Xu; (II) Administrative support: X Xu; (III) Provision of study materials or patients: Z Wang; (IV) \\ Collection and assembly of data: J Zhang; (V) Data analysis and interpretation: J Zhang, S Jiang; (VI) Manuscript writing: All authors; (VII) Final \\ approval of manuscript: All authors. \\ Correspondence to: Xujuan Xu. Nursing Department, Nantong University Affiliated Hospital, School of Nursing, Nantong University, Nantong, \\ China. Email: xxj_1124@126.com.
}

Backgroundk Negative emotions such as anxiety and depression may have a bearing on patients' psychological resilience and quality of life (QoL) following surgery. Cognitive bias modification (CBM) for attention and interpretation has been confirmed to alleviate anxiety and depression. However, the psychological effects of CBM attention and interpretation on Chinese patients with pituitary adenoma have hardly received research attention.

Methods: This prospective interventional study enrolled 100 patients undergoing surgical treatment for pituitary adenoma. Participants were divided into an experimental group and a control group, with 50 cases in each group. Baseline characteristics of included patients were collected. Patients in the control group were given routine training, and those in experimental group received routine training plus CBM attention and interpretation training. The Connor-Davidson Resilience Scale (CD-RISC) was used to assess patients' psychological resilience. The patients' levels of anxiety and depression were assessed using the Self-rating Anxiety Scale (SAS) and Self-rating Depression Scale (SDS). QoL was assessed based on the European Organization for Research and Treatment of Cancer QoL Questionnaire (EORTC QLQ-C30).

Results: No statistical difference was found between the 2 groups in CD-RISC, SAS, SDS, or QoL score at baseline $(\mathrm{P}>0.05)$. After CBM training, psychological resilience and QoL in the experimental group improved, and the levels of anxiety and depression decreased $(\mathrm{P}<0.05)$. Furthermore, the experimental group showed statistically superior psychological resilience and QoL than the control group. The levels of anxiety and depression in the experimental group were statistically lower than those in the control group $(\mathrm{P}<0.05)$.

Conclusions: CBM for attention and interpretation effectively improves the psychological resilience of patients and reduces the symptoms of anxiety and depression, thus improving patients' postoperative QoL.

Trial registration: Chinese Clinical Trial Registry Identifier: ChiCTR2100045899.

Keywords: Cognitive bias modification (CBM); psychological resilience; quality of life (QoL); pituitary adenoma

Submitted Feb 23, 2021. Accepted for publication Apr 23, 2021.

doi: 10.21037/apm-21-782

View this article at: http://dx.doi.org/10.21037/apm-21-782 


\section{Introduction}

Tumors of the anterior pituitary, known as pituitary adenomas, are the most common type of pituitary disorder (1). Pituitary adenomas have shown a steady rise in incidence in China, accounting for $8-15 \%$ of intracranial masses (2). Functional pituitary adenomas can lead to elevated secretion of 1 or multiple hormones, while nonfunctional adenomas compress the areas surrounding the anterior pituitary, resulting in hormonal deficiencies $(3,4)$. Transsphenoidal surgery is the first-line treatment for pituitary adenomas (5). However, surgery entails great psychological pressure as well as physical discomfort, which can deteriorate patients' social psychological state. Therefore, comprehensive surgery-based treatment strategies that include medical therapies, nursing participation, and psychological intervention play an important role in the postoperative treatment of patients with pituitary adenoma $(6,7)$.

The quality of surgical procedures has typically been determined by the rate of survival, complications, and recurrence after surgery, without full consideration being given to the patient's mood or pain associated with the operation. Approximately one-third of cancer patients are reported to suffer from anxiety and depression $(8,9)$. These negative emotions may weaken the patient's psychological resilience and response to positive psychology (10). The implementation of feasible interventions which enhance psychological resilience and reduce negative emotions is vital for improving patients' quality of life (QoL) following surgery. However, psychological intervention has not been investigated in patients with pituitary adenoma in China.

In their study, Mogoase et al. used cognitive bias modification (CBM) for attention and interpretation, targeting systematic modification of biases in the processing of negative information, and confirmed that attention and interpretation biases might play a causal role in the development and maintenance of anxiety (11). Another study showed that successfully reducing threat-related attentional and interpretive biases, and trait and state anxiety through CBM may be beneficial to the prognosis of postoperative patients (12). Firstly, CBM aims to divert the patient's focus more to positive prospects rather than the disease itself, and encourage them to develop a positive, sanguine, and grateful attitude towards life. Secondly, it aims to assist patients to recognize and avoid negative emotions, increase their psychological flexibility, minimize stress, strengthen their emotional management, and cultivate their physical and mental adaptability (13).
In present study, we aimed to determine the effects of CBM on the psychological resilience and QoL of patients after surgery for pituitary adenoma, thereby providing evidence for the use of CBM in these patients. We present the following article in accordance with the CONSORT reporting checklist (available at http://dx.doi.org/10.21037/ apm-21-782).

\section{Methods}

\section{Patient selection}

Adopting the convenience sampling approach, we enrolled 100 patients with pituitary adenoma who were admitted to the Affiliated Hospital of Nantong University between December 2018 and January 2020 into this study. Participants included in this study have provided written informed consent and were divided into 2 groups according to a random number table, an experimental group and a control group, with each group comprising 50 cases. All enrolled patients met the following criteria: (I) aged $\geq 18$ years old; (II) a diagnosis of pituitary adenoma (prolactin-secreting, growth hormone-secreting, adrenocorticotropic hormone-secreting, and thyrotropinsecreting tumors) by biopsy; (III) treated with surgery. Patients participating in other clinical trials were excluded, as were patients who refused to sign a written informed consent form. This study complied with the ethical principles of the Declaration of Helsinki (as revised in 2013) and received approval from the ethics committee of the Affiliated Hospital of Nantong University.

\section{Procedure}

Routine training for patients mainly includes dietary advice, infection prevention, medication guidance, diseaserelated education, and hormone level monitoring. After the operation, a patient exchange meeting was held once a week, for 8 weeks. In addition to routine training, the experimental group received extra CBM training. All patients began their training 1 week after the operation; training sessions lasted for 45 minutes and were held twice a week, for 8 weeks.

\section{CBM for attention}

CBM for attention (CBM-A) was programmed using E-Prime software (14), and consisted of a dot-probe task (15). 
First, a cross pattern appeared on the screen for 1,000 ms. After that, threatening words appeared on the screen 1 by 1 for $750 \mathrm{~ms}$. Then, the words disappeared, and an arrow appeared in the screen, pointing left or right at random. Participants were instructed to press the F key when the arrow pointed left and the J key when the arrow pointed right. Each test was performed at an interval of 1,000 ms. All words used were obtained from the Chinese Affective Words System (16).

\section{CBM for interpretation}

CBM for interpretation (CBM-I) was programmed using E-Prime software, based on Brosan $(12,14)$. In the training, a word, such as "approving", and a sentence, such as "Your supervisor is discussing your future", would be shown on the screen for 1,000 ms. The combinations could have a positive or negative meaning. Patients judged the relationship between the word and the sentence. A response was considered correct when patients identified positive combinations as being related or negative combinations as being unrelated. The attentional and interpretive training included a range of threatening stimuli covering the major domains of generalized social anxiety. All words and phrases used were obtained from the Chinese Affective Words System (16).

\section{Assessment of psychological resilience}

Patients' psychological resilience was assessed using the Connor-Davidson Resilience Scale (CD-RISC) (17). This 25-item scale covers self-confidence, tenacity, and optimism. Each item is scored from $0-4$, with a score of 0 representing "not true at all" and a score of 4 representing "true all the time". Patients are then given an aggregate score out of 100 points. The higher the score, the better the patient's psychological resilience. In 2017, Wu et al. devised a modified, Chinese version of the scale, which yielded a Cronbach $\alpha$ coefficient of 0.91 (17).

\section{Assessment of negative emotion}

The Self-rating Anxiety Scale (SAS) and the Self-rating Depression Scale (SDS) were used to assess patients' anxiety and depression levels $(18,19)$. The SAS and SDS each contain 20 items, and each item is given a score from 1-4 as follows: "none/insignificant" =1 point, "mild/some of the time in frequency" $=2$ points, "moderate/a good part of the time in frequency" $=3$ points, and "severe/most or all of the time in frequency" $=4$ points. An SAS score $\geq 50$ points indicates anxiety, and an SDS score $\geq 53$ points indicates depression. The higher the score, the more severe the anxiety or depression.

\section{Assessment of QoL}

The QoL of the patients was assessed using the European Organization for Research and Treatment of Cancer QoL Questionnaire (EORTC QLQ-C30) within 3 months after discharge from hospital (20). The EORTC QLQ-C30 comprises 30 items, dividing into 5 functional scales (physical, role, emotional, cognitive, and social function). Patients are then given an aggregate score out of 100 points. The higher the overall score, the better the patient's QoL. In the present study, a total score of 75 points was set as the critical value; a total score of $<75$ points indicated a poor QoL, or a score $>75$ points indicated a satisfactory QoL.

\section{Statistical analysis}

Statistical analyses were performed using SPSS (version 22.0, SPSS Inc., Chicago, IL, USA). Continuous variables were reported as means \pm standard deviations, and the $t$ test was used for comparisons between the 2 groups. Categorical variables were reported as numbers and percentages, and the chi-square test was used for comparisons between the 2 groups. The paired t test was used to compare the scores of both groups before and after intervention. For all analyses, $\mathrm{P}<0.05$ was considered to show a significant difference.

\section{Results}

\section{Comparison of general patient information}

This study included 100 postoperative patients with pituitary adenoma, who were divided evenly into the experimental group and control group (Figure 1). In the control group, there were 28 males and 22 females, most of whom $(58 \%)$ were aged between 30 and 50 years old. Only $11(22 \%)$ patients received the education of high school or below, and 38 (76\%) patients were employed at the time of assessment. Most patients had the capacity to pay for their surgical treatment. Of the 4 common types of pituitary adenomas, there were 27 (54\%) prolactin-secreting tumors, 15 (30\%) growth hormone-secreting tumors, 7 (14\%) adrenocorticotropic hormone-secreting tumors, and $1(2 \%)$ thyrotropin-secreting tumor in the control group. The 


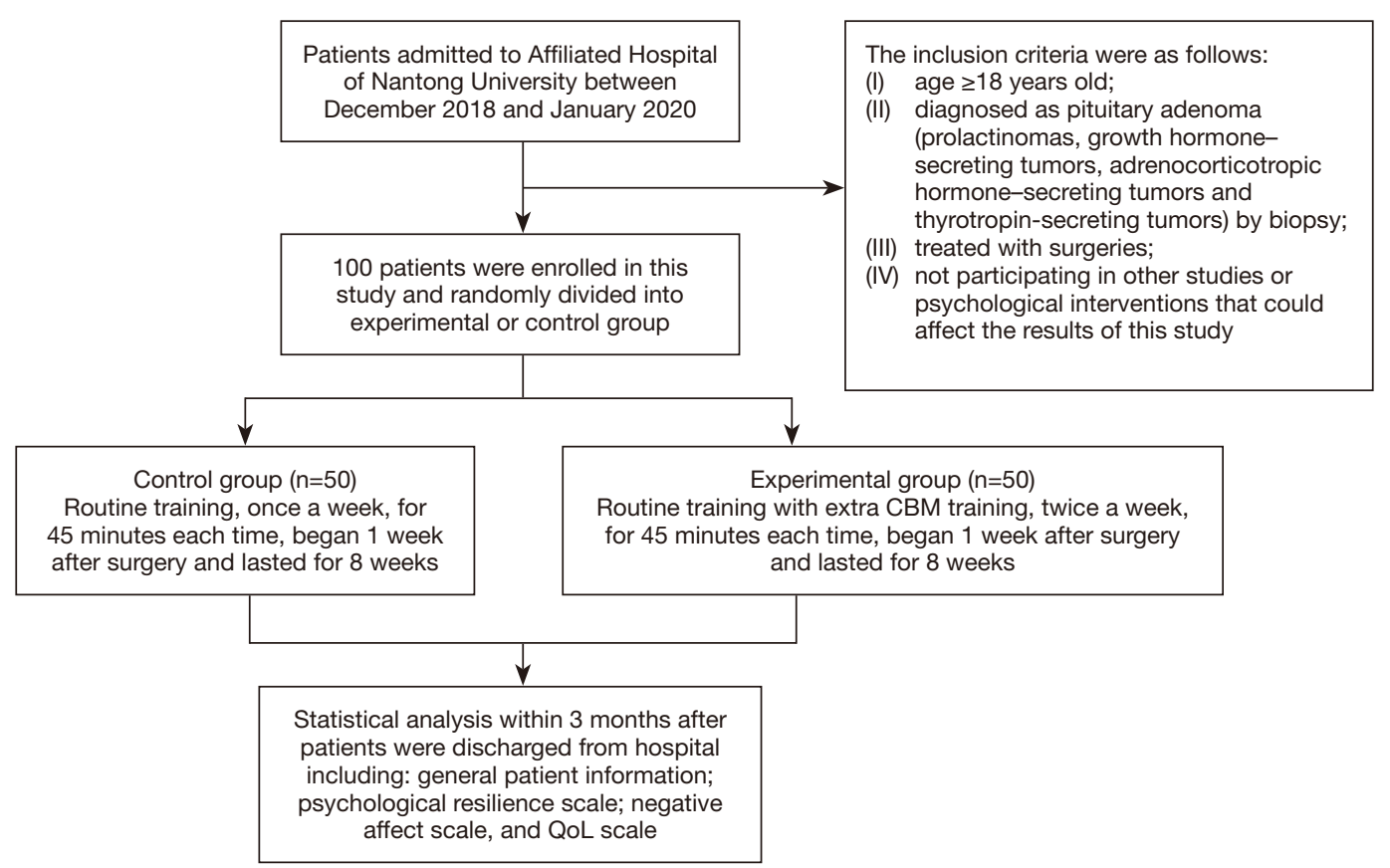

Figure 1 Flow chart of this study. QoL, quality of life.

pituitary adenomas ranged in size from $0.5-8 \mathrm{~cm}$, with most of them measuring between $1-4 \mathrm{~cm}(86 \%)$.

The experimental group comprised 27 males and 23 females, most of whom (60\%) were aged between 30 and 50 years old. Seventeen (34\%) patients received the education of high school or below, and $38(76 \%)$ patients were married at the time of assessment. In the experimental group, there were 31 (62\%) prolactin-secreting tumors, 13 (26\%) growth hormone-secreting tumors, and 6 (12\%) adrenocorticotropic hormone-secreting tumors; none of the patients had a thyrotropin-secreting tumor. There were no statistically significant differences in sex, age, marital status, education level, employment, medical payment method, pituitary adenoma type, or tumor size between the 2 groups ( $\mathrm{P}>0.05$ ), meaning the 2 groups were comparable (Table 1 ).

\section{Changes in psychological resilience scale score}

Before training, the total score and scores for tenacity dimension, self-strength dimension and optimism dimension of the psychological scale showed no statistical difference between the experimental group $(53.48 \pm 6.53$ points, $26.34 \pm 4.69$ points, $17.48 \pm 2.79$ points, and $9.66 \pm 3.56$ points, respectively) and the control group $(54.02 \pm 5.60$ points, $26.62 \pm 3.68$ points, $18.26 \pm 2.90$ points, and $9.14 \pm 3.12$ points, respectively) $(\mathrm{P}>0.05)$. After training, the scores of the experimental group showed a marked increase (62.24 \pm 5.18 points, $29.70 \pm 4.31$ points, $20.56 \pm 1.74$ points, and $11.98 \pm 2.38$ points, respectively) and were significantly higher than those before training $(\mathrm{P}<0.05)$. There was no statistical difference in the pre- and post-training scores in the control group $(\mathrm{P}>0.05)$ (Table 2).

\section{Changes in negative affect scores}

The SAS and SDS scores before and after the intervention are displayed in Table 3. Before training, no statistically significant difference existed between the 2 groups $(\mathrm{P}>0.05)$. After training, the scores in the experimental group (43.86 \pm 3.42 points and $42.46 \pm 3.85$ points, respectively) showed a significant decrease, and were lower than those in the control group $(55.48 \pm 3.79$ points and $55.48 \pm 4.19$ points, respectively) $(\mathrm{P}<0.05)$.

\section{Changes in QoL score}

The QoL of pituitary adenoma patients was assessed based on the EORTC QLQ-C30. The average scores of all 6 items are summarized in Table 4. The scores of physical function, role function, emotional function, cognitive function, social function and global health status in the experimental group $(87.98 \pm 12.83$ points, 
Table 1 General information of the enrolled patients (n, \%)

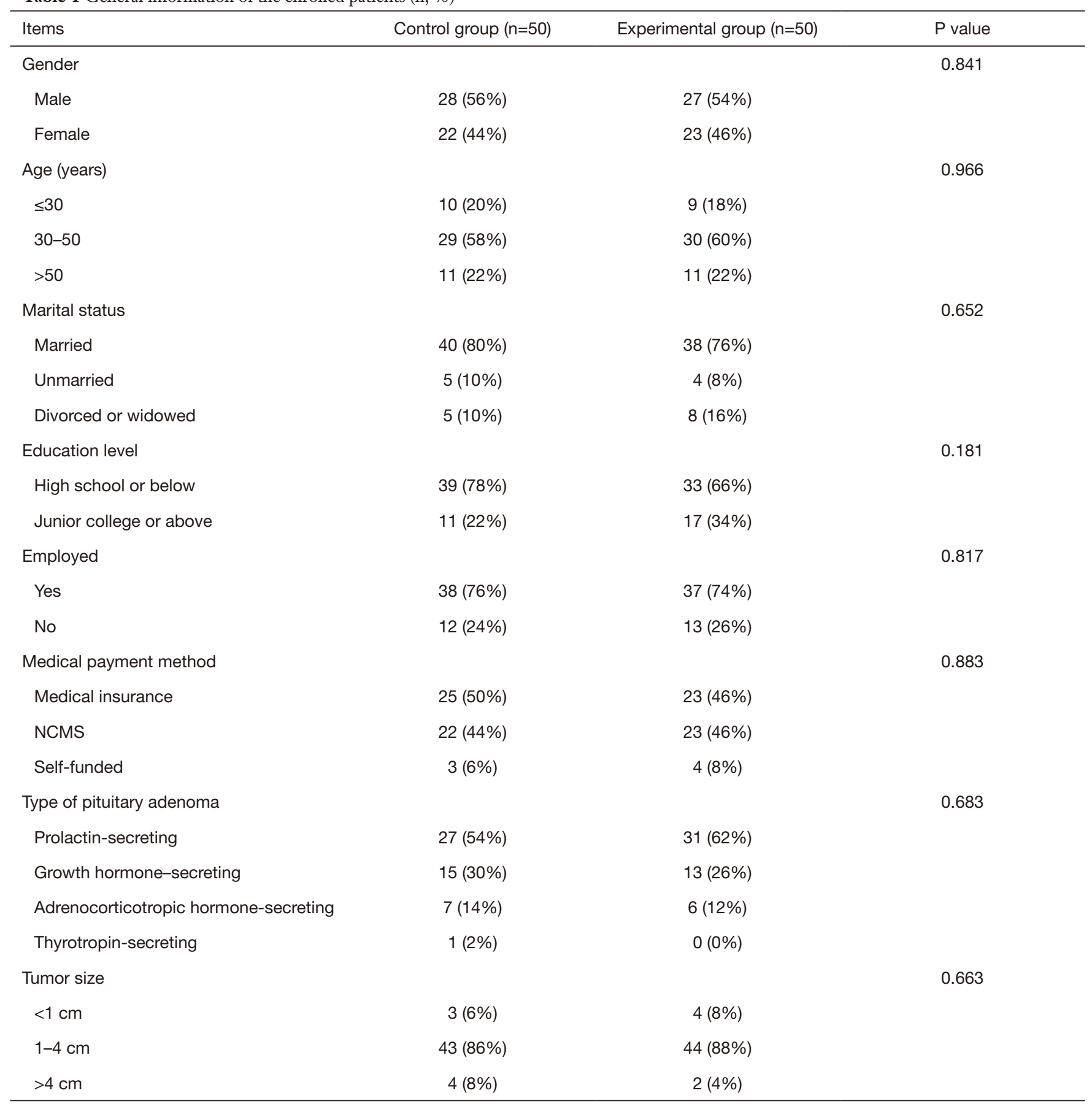

NCMS, New Rural Cooperative Medical Scheme.

$83.32 \pm 15.78$ points, $78.22 \pm 13.22$ points, $94.48 \pm 16.90$ points, $90.04 \pm 17.50$, and $75.52 \pm 13.36$ points, respectively) all increased markedly after CBM training and were significantly higher than those in the control group $(\mathrm{P}<0.05)$. No significant difference was found between the control group's QoL scores before and after training $(\mathrm{P}>0.05)$.

\section{Discussion}

Pituitary adenomas account for $8-15 \%$ of intracranial 
Table 2 Changes in psychological resilience score in the 2 groups before and after CBM $(\bar{x} \pm \mathrm{SD}, \%)$

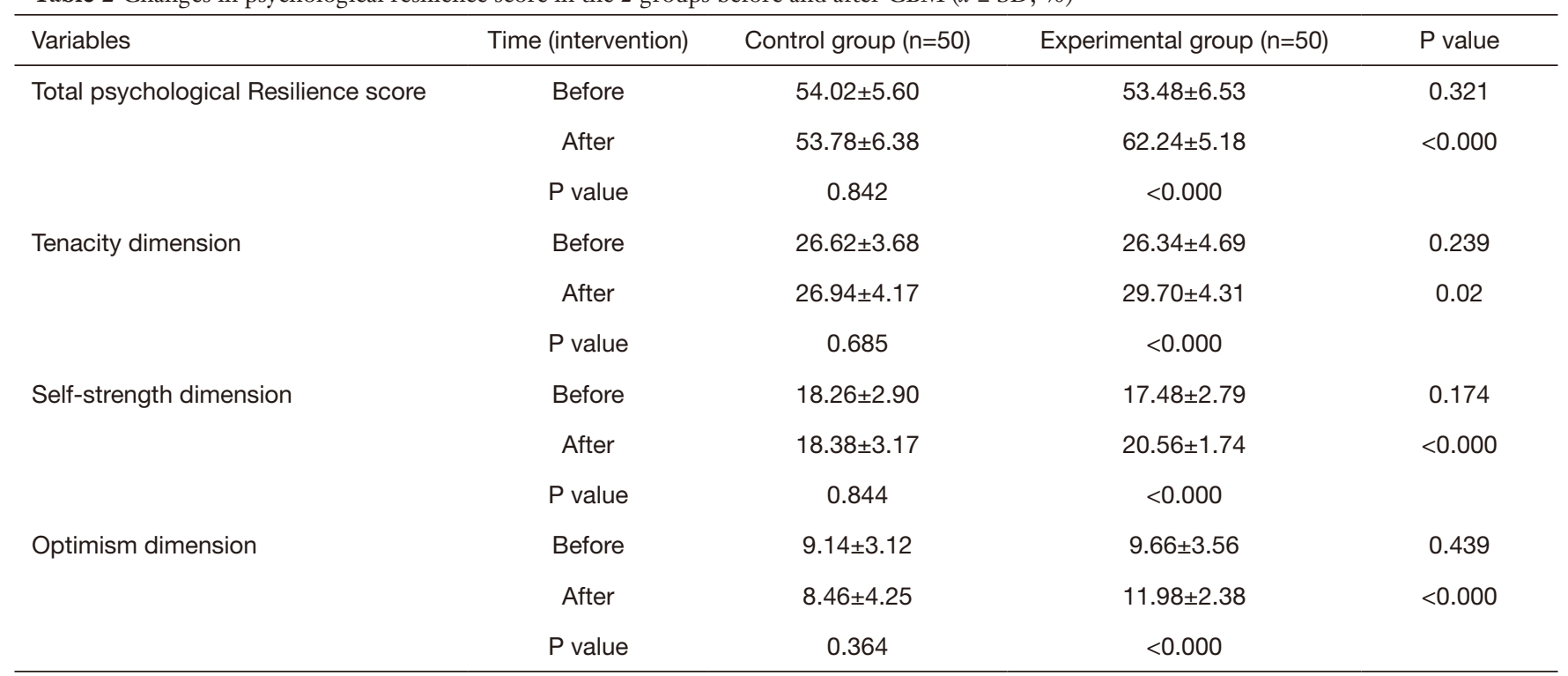

CBM, cognitive bias modification.

Table 3 Changes in SAS and SDS scores in the 2 groups before and after CBM $(\bar{x} \pm \mathrm{SD}, \%)$

\begin{tabular}{|c|c|c|c|c|}
\hline Scale & Time (intervention) & Control group $(n=50)$ & Experimental group $(n=50)$ & $P$ value \\
\hline \multirow{2}{*}{ SAS } & After & $55.48 \pm 3.79$ & $43.86 \pm 3.42$ & $<0.000$ \\
\hline & $P$ value & 0.807 & $<0.000$ & \\
\hline \multirow[t]{2}{*}{ SDS } & Before & $56.10 \pm 4.55$ & $55.90 \pm 4.27$ & 0.821 \\
\hline & $P$ value & 0.480 & $<0.000$ & \\
\hline
\end{tabular}

SAS, Self-rating Anxiety Scale; SDS, Self-rating Depression Scale.

tumors (21). These tumors are mainly treated through a combination of surgery and medicine $(5,22)$. Most patients with pituitary adenoma require long-term drug treatment after undergoing surgery. In addition to the physical damage caused by the disease, patients also face psychological pressures. Patients often feel negative emotions such as anxiety and depression, which can hinder treatment and diminish the QoL. This study found that CBM training can effectively improve the psychological resilience of patients with pituitary adenomas, reduce negative emotions, and improve the QoL.

The results revealed the psychological resilience level in the 2 groups to be lower before than after training. After CBM training, the CDRISC score increased significantly, while that in the control group showed no significant change. This result indicates that CBM-A combined with CBM-I can improve the postoperative resilience of patients with pituitary adenomas by reducing the focus on negative aspects and interpretation bias, which is consistent with the results of similar studies $(12,23,24)$. Participants' attention bias relating to threat in the first session was successfully weakened by training. Participants showed more positive judgment in the interpretation task. These attentional and interpretation biases were accompanied by a significant reduction in trait and state anxiety. Participation in group training and patient seminars has become the best way to encourage patients and to promote their selfunderstanding, change any inappropriate behavior, and enhance their confidence in overcoming the disease. In the non-intervention control group, participants instinctively 
Table 4 Changes of EORTC QLQ-C30 score in the 2 groups before and after CBM $(\bar{x} \pm \mathrm{SD}, \%)$

\begin{tabular}{|c|c|c|c|c|}
\hline Variables & Time (intervention) & Control group $(n=50)$ & Experimental group $(n=50)$ & $P$ value \\
\hline \multirow{2}{*}{ Physical function } & After & $78.94 \pm 13.81$ & $87.98 \pm 12.83$ & 0.001 \\
\hline & $P$ value & 0.565 & $<0.000$ & \\
\hline \multirow[t]{2}{*}{ Role function } & Before & $70.44 \pm 15.36$ & $74.40 \pm 15.02$ & 0.195 \\
\hline & $P$ value & 0.126 & 0.005 & \\
\hline \multirow[t]{3}{*}{ Emotional function } & Before & $64.78 \pm 15.60$ & $67.38 \pm 14.50$ & 0.390 \\
\hline & After & $69.26 \pm 13.90$ & $78.22 \pm 13.22$ & 0.001 \\
\hline & $P$ value & 0.133 & $<0.000$ & \\
\hline Cognitive function & $P$ value & 0.490 & $<0.000$ & \\
\hline \multirow[t]{3}{*}{ Social function } & Before & $83.38 \pm 18.46$ & $80.48 \pm 19.22$ & 0.443 \\
\hline & After & $84.90 \pm 17.65$ & $90.04 \pm 17.50$ & 0.147 \\
\hline & $P$ value & 0.675 & 0.011 & \\
\hline \multirow[t]{3}{*}{ Global health status } & Before & $70.26 \pm 18.00$ & $64.64 \pm 18.04$ & 0.122 \\
\hline & After & $68.22 \pm 16.80$ & $75.52 \pm 13.36$ & 0.018 \\
\hline & $P$ value & 0.559 & 0.001 & \\
\hline
\end{tabular}

EORTC QLQ-C30, European Organization for Research and Treatment of Cancer QoL Questionnaire; CBM, cognitive bias modification.

focused on the disease itself, which increased the related stress and negative emotions they experienced.

The high negative affect scores of the 2 groups before intervention indicated that the patients were in a state of anxiety and depression. After CBM training, the SAS and SDS scores in the experimental group were significantly lower, while the scores of the control group did not show a significant change. At the same time, it was found that after the CBM training, the QoL score of the experimental group was markedly increased, while that in the control group showed no significant change. Depression and anxiety are the main risk factors affecting the QoL of patients, especially their functional state, which is consistent with the findings of $\mathrm{Wu}$ et al. (25). The QoL is usually worse for patients with depression than for those without. Our results suggest that CBM can reduce the anxiety and depression levels of patients following pituitary adenoma surgery and improve their QoL. In practice, the chief problem facing most patients with pituitary adenomas after surgery is longterm drug treatment, which entails an economic burden and time cost, as well as the risk of disease recurrence $(21,22)$. The QoL of patients is affected by their worries about finances and the consequences of stopping taking medicine. In the experimental group, the correction of attention and interpretation biases made participants willing to share their disease-related experiences, pay more attention to peer support, express their inner feelings, and have positive expectations for the future. Through this intervention, the occurrence of anxiety and depression was effectively reduced and the QoL improved.

This study has some limitations. First, the sample size was small, and a larger sample size is needed to verify our results. Furthermore, the study cycle was limited to an 8 -week intervention period; thus, the effects of long-term intervention are still unclear, and a longer study time is required for further research.

\section{Conclusions}

CBM for attention and interpretation effectively improves 
the psychological resilience of patients and reduces their symptoms of anxiety and depression, thus improving their QoL after surgery.

\section{Acknowledgments}

Funding: None.

\section{Footnote}

Trial Protocol: Available at http://dx.doi.org/10.21037/apm21-782

Reporting Checklist: The authors have completed the CONSORT reporting checklist. Available at http://dx.doi. org/10.21037/apm-21-782

Data Sharing Statement: Available at http://dx.doi. org/10.21037/apm-21-782

Conflicts of Interest: All authors have completed the ICMJE uniform disclosure form (available at http://dx.doi. org/10.21037/apm-21-782). The authors have no conflicts of interest to declare.

Ethical Statement: The authors are accountable for all aspects of the work in ensuring that questions related to the accuracy or integrity of any part of the work are appropriately investigated and resolved. This study complied with the ethical principles of the Declaration of Helsinki (as revised in 2013) and received approval from the ethics committee of the Affiliated Hospital of Nantong University. Participants included in this study have provided written informed consent

Open Access Statement: This is an Open Access article distributed in accordance with the Creative Commons Attribution-NonCommercial-NoDerivs 4.0 International License (CC BY-NC-ND 4.0), which permits the noncommercial replication and distribution of the article with the strict proviso that no changes or edits are made and the original work is properly cited (including links to both the formal publication through the relevant DOI and the license). See: https://creativecommons.org/licenses/by-nc-nd/4.0/.

\section{References}

1. Famini P, Maya MM, Melmed S. Pituitary magnetic resonance imaging for sellar and parasellar masses: tenyear experience in 2598 patients. J Clin Endocrinol Metab 2011;96:1633-41.

2. Cox L, Nelson H, Lockey R, et al. Allergen immunotherapy: a practice parameter third update. J Allergy Clin Immunol 2011;127:S1-55.

3. Daly AF, Rixhon M, Adam C, et al. High prevalence of pituitary adenomas: a cross-sectional study in the province of Liege, Belgium. J Clin Endocrinol Metab 2006;91:4769-75.

4. Mazziotti G, Mancini T, Mormando M, et al. High prevalence of radiological vertebral fractures in women with prolactin-secreting pituitary adenomas. Pituitary 2011;14:299-306.

5. Rehman L, Rehman UL, Jabeen R, et al. Endoscopic Trans-Sphenoidal surgery; Efficacy and response in Pituitary Adenoma. Pak J Med Sci 2018;34:412-7.

6. Li Y, Qian Y, Qiao Y, et al. Risk factors for the incidence of apoplexy in pituitary adenoma: a single-center study from southwestern China. Chin Neurosurg J 2020;6:20.

7. Zhu X, Wang Y, Zhao X, et al. Incidence of Pituitary Apoplexy and Its Risk Factors in Chinese People: A Database Study of Patients with Pituitary Adenoma. PLoS One 2015;10:e0139088.

8. Tamler R, Lee AK, Lewis MS, et al. It's all in the head: insomnia and anxiety caused by thyrotropin-secreting pituitary adenoma. Thyroid 2006;16:317-8.

9. Ghanem A, Mouna A, Ben Nasr S, et al. Clinical and therapeutic particularities of depression associated with pituitary adenoma. Tunis Med 2009;87:547-8.

10. Reid R. Psychological Resilience. Med Leg J 2016;84:172-84.

11. Mogoase C, David D, Koster EH. Clinical efficacy of attentional bias modification procedures: an updated metaanalysis. J Clin Psychol 2014;70:1133-57.

12. Brosan L, Hoppitt L, Shelfer L, et al. Cognitive bias modification for attention and interpretation reduces trait and state anxiety in anxious patients referred to an outpatient service: results from a pilot study. J Behav Ther Exp Psychiatry 2011;42:258-64.

13. Lin C, Diao Y, Dong Z, et al. The effect of attention and interpretation therapy on psychological resilience, cancerrelated fatigue, and negative emotions of patients after colon cancer surgery. Ann Palliat Med 2020;9:3261-70.

14. Schneider W, Eschman A, Zuccolotto A. E-prime user's guide. Pittsburgh: Psychology Software Tools, Inc., 2002.

15. MacLeod C, Rutherford E, Campbell L, et al. Selective attention and emotional vulnerability: assessing the 
causal basis of their association through the experimental manipulation of attentional bias. J Abnorm Psychol 2002;111:107-23.

16. Wang Yi-Niu ZL-M, Luo Yue-Jia. The Pilot Establishment and Evaluation of Chinese Affective Words System. Chinese Mental Health 2008;22:608-12.

17. Wu L, Tan Y, Liu Y. Factor structure and psychometric evaluation of the Connor-Davidson resilience scale in a new employee population of China. BMC Psychiatry 2017; 17:49.

18. Zung WW. A rating instrument for anxiety disorders. Psychosomatics 1971;12:371-9.

19. Nishina Y, Yoshioka SI. A Survey of Epilepsy-related Knowledge, Attitudes and Practices of Home Healthcare Nurses in the San-in Region of Japan. Yonago Acta Med 2018;61:19-26.

20. Aaronson NK, Ahmedzai S, Bergman B, et al. The European Organization for Research and Treatment of Cancer QLQ-C30: a quality-of-life instrument for use in international clinical trials in oncology. J Natl Cancer Inst
1993;85:365-76.

21. Lake MG, Krook LS, Cruz SV. Pituitary adenomas: an overview. Am Fam Physician 2013;88:319-27.

22. Molitch ME. Diagnosis and Treatment of Pituitary Adenomas: A Review. JAMA 2017;317:516-24.

23. Nejati V, Fathi E, Shahidi S, et al. Cognitive training for modifying interpretation and attention bias in depression: Relevance to mood improvement and implications for cognitive intervention in depression. Asian J Psychiatr 2019;39:23-8

24. Naim R, Kivity Y, Bar-Haim Y, et al. Attention and interpretation bias modification treatment for social anxiety disorder: A randomized clinical trial of efficacy and synergy. J Behav Ther Exp Psychiatry 2018;59:19-30.

25. Wu Y, Zhang Y, Zou L. Effects of anxiety, depression, and fatigue on quality of life in early esophageal cancer patients following endoscopic submucosal dissection. Ann Palliat Med 2020;9:3766-75.

(English Language Editor: J. Reynolds)
Cite this article as: Zhang J, Xu X, Wang Z, Jiang S. The effects of cognitive bias modification for attention and interpretation on the postoperative psychological resilience and quality of life of patients with pituitary adenoma: a randomized trial. Ann Palliat Med 2021;10(5):5729-5737. doi: 10.21037/apm21-782 